\title{
Déformation $J$-équivalente de polynômes géometriquement finis
}

\author{
par \\ Peter Hä̈sinsky (Bern)
}

\begin{abstract}
Any geometrically finite polynomial $f$ of degree $d \geq 2$ with connected Julia set is accessible by structurally stable sub-hyperbolic polynomials of the same degree. Moreover, they are topologically conjugate to $f$ on their Julia sets.
\end{abstract}

Soit $f: \mathbb{C} \rightarrow \mathbb{C}$ un polynôme de degré $d \geq 2$. Rappelons que l'on définit son ensemble de Julia rempli $K(f)$ comme l'ensemble des points qui ne sont pas attirés par l'infini par itération et son ensemble de Julia par $J(f)=$ $\partial K(f)$.

Soient $C(f)=\left\{f^{\prime}=0\right\}$ son ensemble critique et

$$
\operatorname{Post}(f)=\bigcup_{c \in C(f)} \overline{\bigcup_{n \geq 1}\left\{f^{n}(c)\right\}}
$$

son ensemble postcritique.

DÉfinition. Un polynôme de degré $d \geq 2$ est dit géométriquement fini si l'une des conditions équivalentes suivantes est satisfaite :

(i) l'ensemble postcritique est fini sur l'ensemble de Julia,

(ii) tout point critique est soit prépériodique, soit attiré par un cycle attractif ou parabolique.

En particulier, tous ses cycles indifférents sont paraboliques (le $\S 1$ rappelle les notions liées aux points périodiques dont nous aurons besoin). Un polynôme géométriquement fini sans point parabolique est dit soushyperbolique. On rappelle que l'ensemble de Julia connexe d'un polynôme géométriquement fini est toujours localement connexe ([6]). Il s'ensuit qu'il existe un lacet de Carathéodory $\gamma: \mathbb{T} \rightarrow J_{f}$ continu surjectif tel que $\gamma(d t)=$ $f(\gamma(t))$.

2000 Mathematics Subject Classification: 37F45, 30D05.

Ce travail a été effectué à l'ENS de Lyon, UMPA, CNRS UMR 5669. 
On montre :

ThÉORÈmE. Soit $f: \mathbb{C} \rightarrow \mathbb{C}$ un polynôme de degré $d \geq 2$ géométriquement fini et d'ensemble de Julia connexe (et localement connexe). Il existe $\eta_{0}>0$, une perturbation $\left(f_{\eta}\right)_{\eta \in\left[0, \eta_{0}\right]}$ de polynômes sous-hyperboliques pour $\eta>0$, avec $f_{0}=f$, et un homéomorphisme $\chi_{\eta}: J_{\eta} \rightarrow J_{f}$ tels que $\chi_{\eta} \circ \gamma_{\eta}=\gamma_{f}$ et donc qui conjugue $f_{\eta} \grave{a} f$.

Esquisse de la démonstration. On utilise l'hypothèse géométriquement finie pour construire un déploiement de $f$ d'applications à allure polynomiale qui éclate les points paraboliques en cycles répulsifs de même période et en cycles attractifs en nombre égal à celui des pétales sans changer les relations critiques (Proposition 3.2). Cette construction s'appuie sur une étude de perturbation locale d'une dynamique parabolique faite au $\S 2$.

En reprenant la démonstration de A. Douady et J. H. Hubbard du théorème de redressement, on montre que cette famille est conjuguée à une famille analytique de polynômes structurellement stable (Corollaire 4.3). Ces polynômes correspondent aussi à un déploiement de $f$. Ensuite, grâce à la stabilité de nos polynômes et des points répulsifs en général, on vérifie que les classes d'équivalences induites par les lacets de Carathéodory sont identiques, ce qui termine la démonstration $(\S 5)$.

Notes. 1. La méthode utilisée pour déformer $f$ correspond à un raffinement de celle de A. Douady pour trouver la borne optimale des cycles nonrépulsifs. M. Shishikura utilise aussi des méthodes de chirurgie pour obtenir "ses inégalités" dans le cas des fractions rationnelles (sa construction dans [14] est explicite et le lecteur intéressé est conseillé de la consulter). Cependant, ces deux traitements transforment les points paraboliques en points attractifs sans toujours garder des ensembles de Julia homéomorphes. Notre déformation est donc différente. Une autre approche serait de travailler dans l'espace des polynômes de degré $d$, naturellement paramétrés par $\mathbb{C}^{d-1}$, pour montrer que l'on peut "tuer" tous les points paraboliques. Cependant, il ne semble pas évident à l'auteur de montrer que la déformation peut être faite de manière à imposer la nature des points périodiques résultants.

2. Ce théorème résoud partiellement une conjecture de L. R. Goldberg et J. Milnor ([8]) qui affirme que tout polynôme avec un point parabolique peut être perturbé de manière à transformer ce point en points hyperboliques, et ce, sans changer la topologie ni la dynamique de l'ensemble de Julia. Une autre approche, plus sophistiquée, est donnée dans [3].

3. Ce résultat est tiré de [9].

4. Dans [10], on montre la réciproque dans un cadre plus général que géométriquement fini : étant donné un cycle attractif et un point répulsif de période divisant celle du cycle attractif au bord de son bassin immédiat, il existe un polynôme $g$ et un homéomorphisme $\varphi$ du plan qui transforme 
le point répulsif en point parabolique, le bassin attractif devient un bassin parabolique et $\varphi$ fournit une conjugaison des dynamiques sur les ensembles de Julia. D'autre part, cet homéomorphisme est un $\mu$-homéomorphisme au sens de G. David ([4]).

5. Les résultats de dynamique utilisés sont démontrés e.g. dans $[2,6]$ et $[13]$.

Dans la suite, on supposera toujours que les polynômes sont géométriquement finis et moniques centrés, i.e. tangents à $z \mapsto z^{d}$ à l'infini.

Remerciements. Je remercie A. Douady, C. Petersen et M. Shishikura pour leurs commentaires et les discussions fructueuses que j'ai eues avec eux à ce sujet. Je remercie aussi le rapporteur pour m'avoir aidé à clarifier cet article ainsi que pour m'avoir suggéré des démonstrations alternatives.

1. Dynamique au voisinage d'un point fixe. Soit $f: \overline{\mathbb{C}} \rightarrow \overline{\mathbb{C}}$ une fraction rationnelle de degré $d \geq 2$. On suppose que 0 est un point $k$ périodique, et que sur un voisinage $\Omega$ de l'origine, $g(z):=f^{k}(z)=\lambda z+o(z)$, $\lambda \in \mathbb{C}$. On classifie les germes périodiques par leur multiplicateur, i.e. $\lambda=\left(f^{k}\right)^{\prime}(0)$ en ce cas. Nous décrivons ici la nature des points qui nous intéresse, à savoir répulsif, (super-)attractif et parabolique.

Points répulsifs et attractifs. L'origine est un point répulsif si $|\lambda|>1$ et attractif si $0<|\lambda|<1$.

D'après un théorème de G. Koenigs, il existe une unique application conforme $\varphi: \Omega \rightarrow \mathbb{C}$ telle que

$$
\left\{\begin{array}{l}
\varphi(0)=0, \\
\varphi^{\prime}(0)=1,
\end{array} \quad \text { et } \quad \varphi \circ g(z)=\lambda \varphi(z)\right.
$$

pour $z$ assez de proche de 0 .

Points super-attractifs. Un point est super-attractif si $\lambda=0$. A ce moment, l'application premier retour est de la forme $g(z)=z^{l}+o\left(z^{l}\right), l \geq 2$.

D'après un théorème de Böttcher, il existe une unique application conforme $\varphi: \Omega \rightarrow \mathbb{C}$, appelée la coordonnée de Böttcher, telle que

$$
\varphi \circ g(z)=\varphi(z)^{l}
$$

pour $z$ assez de proche de 0 et $\varphi(0)=0, \varphi^{\prime}(0)=1$.

Le point à l'infini est toujours super-attractif pour un polynôme $f$. Si de plus $f$ est monique et son ensemble de Julia est connexe alors sa coordonnée de Böttcher se prolonge en une application conforme $\varphi_{f}: \overline{\mathbb{C}} \backslash K(f) \rightarrow \overline{\mathbb{C}} \backslash \overline{\mathbb{D}}$ qui vérifie l'équation $(*)$ sur $\overline{\mathbb{C}} \backslash K(f)$. Dire que $K(f)$ est localement connexe est équivalent au fait que l'inverse $\psi_{f}=\varphi_{f}^{-1}$ se prolonge continûment au cercle unité en une application continue surjective $\gamma_{f}: \mathbb{T} \rightarrow J_{f}$, appelée lacet de Carathéodory. 
Points paraboliques. Un point est parabolique si $\lambda$ est une racine de l'unité. Quitte à itérer $f$, on se ramène à un germe de la forme $g(z)=z+z^{\nu+1}+$ $o\left(z^{\nu+1}\right)$.

Un ouvert simplement connexe $\mathcal{P}_{\mathrm{a}} \subset \Omega$ est un pétale attractif si $0 \in \partial \mathcal{P}_{\mathrm{a}}$, $g\left(\overline{\mathcal{P}}_{\mathrm{a}}\right) \subset \mathcal{P}_{\mathrm{a}} \cup\{0\}$ et si $\bigcap_{n \geq 0} g^{n}\left(\overline{\mathcal{P}}_{\mathrm{a}}\right)=\{0\}$. De même, un pétale répulsif est un ouvert simplement connexe $\mathcal{P}_{\mathrm{r}}$ qui est un pétale attractif de $g^{-1}$.

D'après L. Leau et P. Fatou, il existe $\nu$ pétales attractifs disjoints et $\nu$ pétales répulsifs disjoints tels que leur réunion avec 0 est un voisinage de l'origine. Les pétales attractifs et répulsifs sont alternés, et un pétale ne peut intersecter au plus que deux pétales de type opposé. De plus, sur chaque pétale $\mathcal{P}$, l'application $g$ est conjuguée à la translation $z \mapsto z+1$ sur un demi-plan droit si attractif et gauche si répulsif.

2. Perturbation locale d'un point parabolique. Nous travaillons d'abord sur un modèle afin d'établir les propriétés dont nous aurons besoin ultérieurement. On considère une famille d'applications holomorphes

$$
g_{t}(\zeta)=\lambda_{t}(\zeta)+h(\zeta, t)
$$

définies au voisinage de $\zeta=\infty$ et $t=0$, où

$$
\left\{\begin{array}{l}
\lambda_{t}(\zeta)=(1-t) \zeta+1+O(t) \quad \text { et } \\
h(\zeta, t)=(1+\zeta) O\left(t^{2}\right)+(t+1) o(1 / \zeta)
\end{array}\right.
$$

Dans ce paragraphe, nous montrons :

Proposition 2.1. Il existe $t_{0}>0$ et un ouvert du plan $\left.T \supset\right] 0, t_{0}[$ tels que, pour tout $t \in T$ :

(a) $g_{t}$ a un point fixe attractif $\alpha_{t} \asymp 1 / t$;

(b) si $\psi(t)$ est une fonction continue sur $T \cup\{0\}$ et $\operatorname{Re} \psi(0)$ est assez grande, alors, quitte à réduire $t_{0}$ et $T$,

$$
\lim _{n \rightarrow \infty} g_{t}^{n}(\psi(t))=\alpha_{t}
$$

Démonstration. Pour $|t|$ assez petit, $\lambda_{t}$ a un point fixe équivalent à $1 / t$. De plus, on a

$$
\left|g_{t}(\zeta)-\lambda_{t}(\zeta)\right|<\left|\lambda_{t}(\zeta)-\zeta\right|
$$

si $|\zeta|$ est assez grand et $|\zeta-1 / t|$ est de l'ordre de $1 / t$. On déduit par le théorème de Rouché l'existence d'un point fixe $\alpha_{t} \asymp 1 / t$ pour $g_{t}$.

Sous les mêmes conditions que ci-dessus, on a

$$
\frac{g_{t}(\zeta)-g_{t}\left(\alpha_{t}\right)}{\zeta-\alpha_{t}}=1-t+o(t) .
$$

Par suite, pour $t>0$ assez petit, $\alpha_{t}$ est attractif. 
Posons

$$
w_{t}=\frac{\log \left(\zeta-\alpha_{t}\right)-\log \left(-\alpha_{t}\right)}{\log \left(g_{t}\right)^{\prime}\left(\alpha_{t}\right)},
$$

où on a choisi une détermination du logarithme telle que $\log \left(\mathbb{R}_{+}-\{0\}\right)=\mathbb{R}$ et $t \mapsto w_{t}(\psi(t))$ est continue au voisinage de 0 .

On a $w_{t}(\zeta) \rightarrow \zeta$ quand $t$ tend vers 0 uniformément sur tout compact, et, dans cette coordonnée, $g_{t}$ s'écrit

$$
T_{t}: w \mapsto w+1+O(t)+O(1 / w),
$$

où $O(1 / w)$ est indépendant de $t$.

Pour $t>0$, le point $\alpha_{t}$ est envoyé en "Re $=+\infty$ ", et, si $\operatorname{Re} \psi(t)$ est assez grande, $t$ assez petit, alors $\operatorname{Re} T_{t}^{n} w_{t}(\psi(t))$ tend vers $+\infty$ avec $n$, i.e. $\lim g_{t}^{n}(\psi(t))=\alpha_{t}$. On remarque que l'on retrouve le fait que $\alpha_{t}$ est attractif.

Tout ceci s'étend sans difficulté à un ouvert $T \supset] 0, t_{0}[$.

3. Construction d'un déploiement. Soit $f: \mathbb{C} \rightarrow \mathbb{C}$ un polynôme de degré $d$ d'ensemble de Julia connexe. Sans perte de généralité, on peut supposer que les cycles attractifs sont en fait super-attractifs, qu'il n'y a qu'une seule grande orbite critique dans chaque cycle de Fatou, voire même que deux points critiques dans une même composante sont confondus.

Proposition 3.1. Il existe un polynôme $R$ (de degré élevé) tel que, pour $\eta>0$ assez petit, $f_{\eta}=f+\eta R$ vérifie les propriétés suivantes:

(i) toutes les orbites critiques prépériodiques de $f$ sont des orbites prépériodiques de $f_{\eta}$,

(ii) tout cycle parabolique ayant $p$ cycles de pétales devient un cycle répulsif et s'éclate en $p$ cycles attractifs.

Démonstration. On considère un polynôme $R$ qui vérifie les conditions suivantes :

(a) les orbites critiques finies $\left\{f^{n}(c)\right\}_{n \geq 0}$ sont des racines de $R$ de multiplicité plus grande que celle de $c$ en tant que point critique de $f$,

(b) pour chaque cycle parabolique $\left\{x_{i}\right\}_{1 \leq i \leq k}, x_{i}$ est racine simple et

$$
\sum_{1 \leq i \leq k} \frac{R^{\prime}\left(x_{i}\right)}{f^{\prime}\left(x_{i}\right)}=1
$$

(c) une condition linéaire sur les dérivées d'ordre supérieur de $R$ sur les cycles paraboliques qui sera précisée ultérieurement.

Le polynôme $R$ existe. En effet, les conditions ci-dessus conduisent à un système linéaire en les coefficients de $R$, qui admet une solution si le degré de $R$ est assez grand.

(a) implique (i). La vérification est immédiate, et laissée au lecteur. 
(b) implique que les points paraboliques deviennent répulsifs. Soit $\left\{x_{i}\right\}_{0 \leq i<k}$ un cycle parabolique. C'est encore un cycle périodique de même période. Calculons, pour $\eta>0$, son multiplicateur :

$$
\left(f_{\eta}^{k}\right)^{\prime}\left(x_{0}\right)=\prod_{1 \leq i \leq k}\left(f^{\prime}\left(x_{i}\right)+\eta R^{\prime}\left(x_{i}\right)\right) .
$$

En passant au "log", on obtient

$$
\begin{aligned}
\log \left|\left(f_{\eta}^{k}\right)^{\prime}\left(x_{0}\right)\right| & =\sum_{1 \leq i \leq k} \log \left|f^{\prime}\left(x_{i}\right)\right|+\sum_{1 \leq i \leq k} \log \left|1+\eta \frac{R^{\prime}\left(x_{i}\right)}{f^{\prime}\left(x_{i}\right)}\right| \\
& =\eta \sum_{1 \leq i \leq k} \frac{R^{\prime}\left(x_{i}\right)}{f^{\prime}\left(x_{i}\right)}+O\left(\eta^{2}\right)>0 .
\end{aligned}
$$

Donc le cycle est devenu répulsif.

Définition de la condition (c). Pour simplifier les notations, on suppose que

$$
\left\{\begin{array}{l}
x_{0}=0, \\
f^{k}(z)=\lambda z+o(z), \quad \text { où } \lambda^{q}=1, \\
f^{k q}(z)=z+z^{\nu+1}+o\left(z^{\nu+1}\right), \quad \text { où } \nu=p q \in \mathbb{N}^{*}, \\
R(z)=\left(z-x_{i}\right) R^{\prime}\left(x_{i}\right)+v_{i}(z) \quad \text { au voisinage de } x_{i} .
\end{array}\right.
$$

La condition (c) exprimera le fait que le développement de $f_{\eta}^{k q}$ au voisinage de $x_{0}=0$ sera du type

$$
f_{\eta}^{k q}(z)=f^{k q}(z)+(\eta q) z+\eta \widehat{h}(z, \eta), \quad \text { où } \quad \widehat{h}(z, \eta)=o\left(z^{\nu+1}\right)+O(\eta) .
$$

On considère deux fonctions $g$ et $u$ telles que

$$
\left\{\begin{array}{l}
g(z)=x_{i}+h(z), \quad \text { où } \quad h(z)=O(z) \\
u(z)=O(z)
\end{array}\right.
$$

On a

$$
\begin{aligned}
f_{\eta}(g(z)+\eta u(z))= & f(g(z)+\eta u(z))+\eta R^{\prime}\left(x_{i}\right) h(z)+\eta v_{i}(g(z))+o(\eta), \\
= & f \circ g(z)+\eta f^{\prime}(g(z)) u(z) \\
& +\eta R^{\prime}\left(x_{i}\right) h(z)+\eta v_{i}(g(z))+o(\eta) .
\end{aligned}
$$

Posons

$$
\left\{\begin{array}{l}
f_{\eta}^{i}=f^{i}+\eta u_{i}(z)+o(\eta) \\
g=f^{i}
\end{array}\right.
$$

On trouve que

$$
u_{i+1}(z)=f^{\prime}\left(f^{i}(z)\right) u_{i}(z)+v_{i}\left(f^{i}(z)\right)+R^{\prime}\left(x_{i}\right)\left(f^{i}(z)-x_{i}\right) .
$$

La condition (c) s'énonce ainsi

$$
u_{k q}(z)=q z+o\left(z^{\nu+1}\right) .
$$


Notez que le terme linéaire provient de la condition (b), i.e. de

$$
\left(f_{\eta}^{k q}\right)^{\prime}(0)=(1+\eta+o(\eta))^{q}=1+\eta q+o(\eta) .
$$

On constate que $(\mathrm{c})$ conduit à un système linéaire en les dérivées successives de $R$ en chaque point de chaque cycle parabolique, comme annoncé ci-dessus.

(b) et (c) implique (ii). Vérifions maintenant que, pour $\eta>0$ assez petit, on a bien création de $p=\nu / q$ cycles attractifs : lorsque $\eta$ est non nul, on a création de $\nu$ points fixes de $f_{\eta}^{k q}$, qui sont en fait classés en $p$ cycles de longueur $q$ de $f_{\eta}^{k}$ (d'après le principe de l'argument). En faisant un changement de variable en $\zeta=-1 /\left(\nu z^{\nu}\right)$, on conjugue $f_{\eta}^{k q}$ à

$$
\zeta \mapsto(1-\nu \eta) \zeta+(1-(\nu+1) \eta)+O(1 / \zeta)+\eta O\left(1 /|\zeta|^{1+1 / \nu}\right)+o(\eta)
$$

au voisinage de l'infini. Nous sommes donc en mesure d'appliquer la Proposition 2.1(a).

Pour $\eta \in \mathbb{C}$, on pose $f_{\eta}=f+\eta R$. Le résultat en vue est :

Proposition 3.2. Il existe $\eta_{0}>0$, un ouvert $\left.\left.S \supset\right] 0, \eta_{0}\right]$ et des ouverts $\left(U_{\eta}^{\prime}, U\right)_{\eta \in S}$ tels que $\left(U_{\eta}^{\prime} \stackrel{f_{\eta}}{\longrightarrow} U\right)_{\eta \in S}$ soit une famille analytique d'applications à allure polynomiale de degré $d$. De plus, les cycles paraboliques ont été perturbés en un cycle répulsif, et en cycles attractifs (en nombre égal à celui des cycles de pétales).

D'après [7], une application à allure polynomiale est la donnée d'un triplet $\left(U^{\prime}, U, f\right)$ où $U^{\prime} \subset \subset U$ sont des disques topologiques et $f: U^{\prime} \rightarrow U$ est une application holomorphe propre de degré $d \geq 2$. On définit alors son ensemble de Julia rempli par $K(f)=\bigcap_{n \geq 0} f^{-n}(\bar{U})$.

La famille d'applications à allure polynomiale $\left(U_{\eta}^{\prime} \stackrel{f_{\eta}}{\longrightarrow} U\right)_{\eta \in S}$ est dite analytique si la dépendance en $\eta$ est analytique.

Démonstration (de la Proposition 3.2). On note $U$ un disque bordé par une équipotentielle de $f$ de très grande valeur, et pour tout $\eta \in \mathbb{D}, U_{\eta}^{\prime}=$ $f_{\eta}^{-1}(U)$. L'image par $f$ de $\partial U^{\prime}$ fait $d$ fois le tour de $\partial U$, donc si $\eta$ est assez petit, il en sera de même pour le déploiement : du coup, $\left(U_{\eta}^{\prime} \stackrel{f_{\eta}}{\longrightarrow} U\right)_{|\eta|<\eta_{0}}$ est une famille analytique d'applications à allure polynomiale de degré $d$.

La Proposition 3.1 nous assure la création d'un point attractif dans chaque pétale pour $\eta>0$ assez petit. La condition d'être attractif étant une condition ouverte, l'ouvert $S$ de l'énoncé existe donc.

4. Une propriété de continuité. L'intérêt des applications à allure polynomiale est qu'elles sont conjuguées à des polynômes ([7], Théorème 1, p. 296; voir aussi la démonstration du Corollaire 4.3) : 
ThÉorème 4.1 De Redressement (A. Douady \& J. H. Hubbard). Soit $\left(U^{\prime}, U, f\right)$ une application à allure polynomiale de degré $d \geq 2$. Il existe un polynôme $P$ de même degré et un homéomorphisme quasiconforme $\varphi: U \rightarrow$ $\varphi(U)$ tels que $\varphi \circ f=P \circ \varphi, \varphi(K(f))=K(P)$ et $\partial_{\bar{z}} \varphi=0$ pp. sur $K(f)$.

Pour la définition et les propriétés des applications quasiconformes, on peut se reporter à $[1]$.

Proposition 4.2. La famille $\left\{f_{\eta}\right\}_{\eta \in S}$ est structurellement stable.

Une famille analytique d'applications à allure polynomiale $\left(f_{\lambda}\right)_{\lambda \in \Lambda}$ est structurellement stable si, étant donné $\lambda_{0} \in \Lambda$, il existe une famille analytique d'homéomorphismes $\left(\chi_{\lambda}\right)$ qui conjuguent $f_{\lambda_{0}}$ à $f_{\lambda}$ au voisinage de leurs ensembles de Julia. D'après [11], c'est le cas si le nombre de cycles attractifs est constant.

Démonstration (de la Proposition 4.2). Nous allons donc montrer que le nombre de cycles attractifs est constant sur $S$, ce qui montrera la proposition. Pour cela, il suffit de vérifier que les points critiques qui étaient attirés par un cycle parabolique le sont maintenant par un cycle attractif qu'il a créé. En effet, un cycle attractif attire toujours un point critique; mais comme ceux de $f$ sont persistants par la déformation (Proposition 3.1), on en déduit d'une part qu'aucun ne disparaît et d'autre part que les seuls créés ne peuvent attirer que les points critiques d'un bassin parabolique. Rappelons que chaque cycle de pétale attractif contient au moins une orbite critique.

Considérons donc un point critique $c$ et un point $k$-périodique parabolique $\alpha$ tel que $f^{k n}(c) \rightarrow \alpha$. Si celui-ci est de nombre de rotation $p / q$, alors $f^{k q n}(c)$ appartient à un même pétale attractif pour $n$ assez grand. Lorsque l'on fait la perturbation, ce point est attiré par le nouveau cycle attractif créé situé dans ce pétale d'après la Proposition 2.1(b).

En comptant les points critiques, on conclut qu'il ne peut s'en créer davantage.

Corollaire 4.3. Il existe une famille analytique de polynômes $\left(P_{\eta}\right)_{\eta \in S}$ de degré $d$ et des homéomorphismes quasiconformes $\phi_{\eta}: \overline{\mathbb{C}} \rightarrow \overline{\mathbb{C}}, \eta \in S$, qui vérifient les propriétés suivantes:

(i) $\phi_{\eta}$ conjugue $f_{\eta} \grave{a} P_{\eta}$ sur un voisinage de leurs ensembles de Julia remplis;

(ii) $\phi_{\eta}$ est holomorphe à l'intérieur de $K\left(f_{\eta}\right)$;

(iii) $\phi_{\eta} \rightarrow$ id et $P_{\eta} \rightarrow f$ quand $\eta \rightarrow 0$.

Dém o n stration. Reprenons la démonstration du Théorème 4.1 dans notre cas. 
Soit $\varphi_{f}: \mathbb{C} \backslash K_{f} \rightarrow \mathbb{C} \backslash \overline{\mathbb{D}}$ la coordonnée de Böttcher de $f$; on a $\varphi_{f}(\partial U)=$ $(|z|=R)$. Notons $\psi_{f}=\varphi_{f}^{-1}$. On définit $\tau_{\eta}:\left(|z|=R^{1 / d}\right) \rightarrow \partial U_{\eta}^{\prime}$ par $f_{\eta} \circ \tau_{\eta}(z)=\psi_{f}\left(z^{d}\right)$, pour $\eta$ appartenant à un voisinage de l'origine. On normalise $\tau_{\eta}$ de façon que $\eta \mapsto \tau_{\eta}$ soit continue et que $\tau_{0}=\psi_{f}$. Nous laissons au lecteur le soin de déterminer une extension lisse $\Psi_{\eta}$ de $\tau_{\eta}$ et $\psi_{\eta}$ en des difféomorphismes de manière continue et telle que les coefficients de Beltrami tendent uniformément vers 0 avec $\eta$. On a alors $\Psi_{0}=\psi_{f}$.

De plus, ceci permet de prolonger $f_{\eta}: U_{\eta}^{\prime} \rightarrow U$ à $\overline{\mathbb{C}}$ en un revêtement ramifié de degré $d$ en posant

$$
\begin{cases}f_{\eta}(z)=\psi_{f}\left(\Psi_{\eta}^{-1}(z)^{d}\right) & \text { si } z \in U-U_{\eta}^{\prime} \\ \psi_{f}\left(\varphi_{f}(z)^{d}\right)=f(z) & \text { si } z \notin U\end{cases}
$$

Soient $\mu_{0}$ la forme de Beltrami induite par la structure complexe standard, et $\mu_{\eta}=\left(\Psi_{\eta}\right)_{*} \mu_{0}$; alors $\left\|\mu_{\eta}-\mu_{0}\right\|_{\infty}$ tend vers zéro avec $\eta$ sur tout compact de $U \backslash \overline{U^{\prime}}$. On prolonge les $\mu_{\eta}$ à $\overline{\mathbb{C}}$ par $\left(f_{\eta}^{n}\right)^{*} \mu_{\eta}, n \geq 1$, et sinon par $\mu_{0}$. On a donc une famille de formes de Beltrami uniformément bornée, à support dans un compact fixe et invariantes par $f_{\eta}$. Il s'ensuit que, pour tout $\eta$, il existe une unique application quasiconforme $\phi_{\eta}: \overline{\mathbb{C}} \rightarrow \overline{\mathbb{C}}$ qui intègre $\mu_{\eta}$ et qui est tangente à l'identité à l'infini (théorème d'intégration des formes de Beltrami). Par construction, $\phi_{\eta}$ conjugue $f_{\eta}$ à un polynôme $P_{\eta}$.

De plus, les formes de Beltrami tendent vers 0 avec $\eta$ car c'est le cas de celles de $\Psi_{\eta}$. Par compacité des applications quasiconformes de dilatation uniformément bornée et unicité des applications quasiconformes normalisées avec formes de Beltrami prescrites, on en déduit que $\phi_{\eta}$ converge vers id. Par conséquent, $P_{\eta}=\phi_{\eta} \circ f_{\eta} \circ \phi_{\eta}^{-1}$ tend vers $f$ quand $\eta \rightarrow 0$.

La famille étant structurellement stable, $f_{\eta}$ dépend analytiquement des multiplicateurs des cycles attractifs créés, et donc $P_{\eta}$ aussi puisque $\phi_{\eta}$ est holomorphe à l'intérieur des ensembles de Julia remplis (voir [12]).

5. Preuve du théorème. Le déploiement associé à $f$ est composé de polynômes sous-hyperboliques ayant des ensembles de Julia connexes et localement connexes. Leurs lacets de Carathéodory sont donc bien définis.

Pour chaque $\eta$, on définit la relation d'équivalence $\sim_{\eta}$ suivante sur $\mathbb{T}=$ $\mathbb{R} / \mathbb{Z}:$

$$
t \sim_{\eta} t^{\prime} \Longleftrightarrow \gamma_{\eta}(t)=\gamma_{\eta}\left(t^{\prime}\right)
$$

où $\gamma_{\eta}$ est le lacet de Carathéodory de $P_{\eta}$. Selon [5], l'ensemble de Julia $P_{\eta}$ est homéomorphe au quotient $\mathbb{T} / \sim_{\eta}$.

Nous allons montrer que ces classes d'équivalence sont toutes les mêmes : on en déduira alors que les dynamiques sur les ensembles de Julia sont topologiquement conjuguées. 
Comme tous les polynômes sont géométriquement finis il suffit de le vérifier sur les cycles de $J_{f}$.

Si le cycle est répulsif, alors tout rayon qui aboutit à un point de ce cycle continue à y aboutir pour $\eta$ assez petit en vertu de la Proposition 5.1 ci-dessous, et donc sur $S$ par stabilité structurelle.

Proposition $5.1([6])$. Soient $f$ un polynôme et $\theta \in \mathbb{Q} / \mathbb{Z}$. On suppose que $\gamma(\theta)=z_{0} \in J_{f}$ est bien défini, prépériodique répulsif, et qu'aucun $f^{i}\left(z_{0}\right)$ n'est un point critique de $f$ pour aucune valeur de $i \geq 0$. Il existe alors un voisinage $U$ de $f$ et une application holomorphe $z: U \rightarrow \mathbb{C}$ tels que $\forall g \in U, \gamma_{g}(\theta)=z(g)$ est un point prépériodique répulsif de $g$.

S'il s'agit d'un cycle parabolique, on peut appliquer la Proposition 2.1(b) au germe inverse avec $\psi$ un point du rayon. On montre ainsi que le rayon aboutit au point répulsif.

Remarque. Si l'ensemble de Julia de $f$ n'est pas connexe, on peut déterminer un déploiement de polynômes comme dans le paragraphe 4 . Cependant, notre dernier argument ne s'applique plus. Néanmoins, un argument de pull-back - suggéré par le rapporteur, et qui s'applique dans des situations plus générales - permet de montrer que les ensembles de Julia restent homéomorphes. Nous esquissons ici cet argument.

On considère un ouvert $V$ (resp. $V_{\eta}$ ) constitué des pétales attractifs de $f$ (resp. de domaines linéarisables des points attractifs créés, avec les points répulsifs correspondant dans leur bord). On définit alors un homéomorphisme $\varphi$ tel que :

- $\varphi$ conjugue $f$ à $P_{\eta}$ au voisinage des points attractifs,

- $\varphi(V)=V_{\eta}$,

- les orbites critiques de $f$ restreintes à $\overline{\mathbb{C}}-V$ sont transformées en celles de $P_{\eta}$ et la restriction de $\varphi$ à cet ensemble est une conjugaison, et

- sur $\partial V$, on a $\varphi \circ f=P_{\eta} \circ \varphi$.

Ces conditions sont facilement satisfaites si on choisit correctement $V$ et $V_{\eta}$.

On peut alors définir une suite d'homéomorphismes $\left(\varphi_{n}\right)$, qui coïncident avec $\varphi$ sur $V$ et sur les orbites critiques de $\overline{\mathbb{C}}-V$, et sont tels que $\varphi_{n} \circ f=$ $P_{\eta} \circ \varphi_{n+1}$ sur $\overline{\mathbb{C}}-V$. Sur tout compact de $\overline{\mathbb{C}}-J_{f}$, la suite $\left(\varphi_{n}\right)$ est constante à partir d'un certain rang, donc uniformément convergente. Nos polynômes étant géométriquement finis et donc faiblement expansifs sur les ensembles de Julia (voir par exemple [2], $\S 6$, ou [6]), on en déduit que les suites $\left(\varphi_{n}\right)$ et $\left(\varphi_{n}^{-1}\right)$ sont toutes deux globalement uniformément convergentes. Leurs limites conjuguent $f$ à $P_{\eta}$ sur leurs ensembles de Julia. 


\section{Références}

[1] L. Ahlfors, Lectures on Quasiconformal Mappings, Van Nostrand, 1966.

[2] H. Brolin, Invariant sets under iteration of rational functions, Ark. Mat. 6 (1965), 103-144.

[3] G. Cui, Geometrically finite rational maps with given combinatorics, preprint, 1997.

[4] G. David, Solutions de l'équation de Beltrami avec $\|\mu\|_{\infty}=1$, Ann. Acad. Sci. Fenn. Ser. A I Math. 13 (1988), 25-70.

[5] A. Douady, Descriptions of compact sets in $\mathbb{C}$, in: Topological Methods in Modern Mathematics (Stony Brook, NY, 1991), Publish or Perish, Houston, TX, 1993, 429-465.

[6] A. Douady et J. H. Hubbard, Étude dynamique des polynômes complexes I, II, Publ. Math. Orsay 84-02 et 85-05, 1984/85.

[7] - - - On the dynamics of polynomial-like mappings, Ann. Sci. École Norm. Sup. 18 (1985), 287-343.

[8] L. R. Goldberg and J. Milnor, Fixed points of polynomial maps. II. Fixed point portraits, ibid. 26 (1993), 51-98.

[9] P. Haïssinsky, Applications de la chirurgie holomorphe, notamment aux points paraboliques, thèse de l'Université de Paris-Sud, Orsay, 1998.

[10] -, Chirurgie parabolique, C. R. Acad. Sci. Paris 327 (1998), 195-198.

[11] R. Mañé, P. Sad and D. Sullivan, On the dynamics of rational maps, Ann. Sci. École Norm. Sup. 16 (1983), 193-217.

[12] C. T. McMullen and D. P. Sullivan, Quasiconformal homeomorphisms and dynamics III. The Teichmüller space of a holomorphic dynamical system, Adv. Math. 135 (1998), 351-395.

[13] J. Milnor, Dynamics in One Complex Variable: Introductory Lectures, Vieweg, Braunschweig, 1999.

[14] M. Shishikura, On the quasiconformal surgery of rational functions, Ann. Sci. École Norm. Sup. 20 (1987), 1-29.

Universität Bern

Mathematisches Institut

Sidlerstrasse 5

3012 Bern, Schweiz

E-mail: phaissin@math-stat.unibe.ch

Received 25 January 1999;

in revised form 30 September 1999 